\title{
Community Oriented Prevention and Rehabilitation of Burns
}

"Anything that's human is mentionable, and anything that is mentionable can be more manageable. The people around can help us know that we are not alone." Fred Rogers

In spite of the efforts of public health physicians to prevent burn injury through fire prevention, awareness campaigns and educational programs, still burn injuries continue to present a significant social and financial burden through patient mortality, morbidity, and long - term disability. Life after burns is a continuous struggle to improve quality of life in society, seeking employment to remain functional, acceptance in community without any stigmatization and medical support to combat health problems. Cost of medical care and rehabilitation is enormous and can be avoided through community oriented prevention of burns and later on effective rehabilitation in particular community. The causes and types of burn injuries determine prevention and rehabilitation plans. Especially when burns are associated with violence, terrorism conflicts and fights. The conflicts include inter racial differences; inter religious assassinations, revengeful traditions, attempted murders, honor killings, domestic violence and terrorism in a community. ${ }^{1}$

The use of acid to produce facial burns in women was witnessed in many rural communities in developing countries. ${ }^{2}$ The first recorded acid attacks in developing countries occurred in Bangladesh in 1967, followed by India in 1982, and Cambodia in 1993. Since then, research has witnessed an increase in the amount and severity of acid attacks in the region. However, this can be traced to significant underreporting in the 1980s and 1990s, along with a general lack of research for this phenomenon during that period. ${ }^{3}$ Acid attacks were reported in many parts of the world. Since the 1990s, Bangladesh had been reporting the highest number of attacks and highest incidence rates for women with 3,512 Bangladeshi people acid attacked between 1999 and 2013. ${ }^{3}$ Although acid attacks occur all over the world, including in Europe and the United States, this type of gender based violence is concentra- ted in rural communities of India, Bangladesh and Pakistan where the implementation of regulations needs further exploration. In Pakistan, the majority of these attacks occurred in the summer. According to a report, up to 150 attacks on women occurred every year. They also reported that the attacks were often the result in rise of domestic abuse, and the majority of victims were female. ${ }^{4}$ The gender violence increased the morbidity rates in burns survivors.

The efforts to produce community oriented prevention and rehabilitation of the adult burn survivors and to measure their health outcomes, preventing social isolation, providing social support and better quality of life after burn injury are almost negligible. WHO working groups states that quality of life is an individual perception of their position in life in context of culture and values system which they live in relation to their goals, expectations, standard and concerns. ${ }^{5}$ Eventual outcome depends on injury severity, individual physical characteristics of patient's motivation, and social support by family and friends. ${ }^{5}$ It was documented that large number of people living with burn scars need social support more than healthy people for better quality of life. $^{6}$

There is a dire need for better understanding of multifaceted determinants of burn injuries and quality of life in adult survivors in the context of society nor$\mathrm{ms}$ and cultural pressures in community settings, in order to plan better preventive strategies to combat this public health problem. Prevention efforts are urgently needed to reduce the rate of these unacceptably high burn injuries, and should be developed on a local level in response to risk factors identified in individual areas. Community oriented prevention and rehabilitation of burns is a cost effective strategy. Community participation, use of appropriate technology, accessibility and equitable distribution of preventive and rehabilitative services are its components. Thus efforts should be directed to decrease sufferings of the burn survivors in society and social support systems should be developed to improve quality of life of burn victims 
through community oriented prevention and rehabilitation of burns.

\section{References}

1. Aslam M, Niazi MZ, Mustafa, G. Pattern of Burns at Lady Reading Hospital, Peshawar. J Postgrad Med Inst. 2012; 26: 221-5.

2. Fava GA, Grandi S, Rafanelli C, Ruini C, Conti S, Belluardo P. Long - term outcome of social phobia treated by exposure. Psychological Medicine, 2001; 31 (5): 899-905.

3. Forjuoh SN. Burns in low - and middle - income coun- tries: a review of available literature on descriptive epidemiology, risk factors, treatment, and prevention. Burns: journal of the International Society for Burn Injuries, 2006; 32: 529-37.

4. International Women's Rights Action Watch Asia Pacific, 2013. [Internet]. 2016. Available from: https:// castanglobalinterns.wordpress.com/2013/.../internationa l-womens-rights-action.

5. WHO Disease and injury country estimates. World Health Organization. Retrieved Nov 11, 2009.

6. Moi AL, Vindenes HA, Gjengedal E. The experience of life after burn injury: a new bodily awareness. Journal of Advanced Nursing, 2008; 64: 278-286.

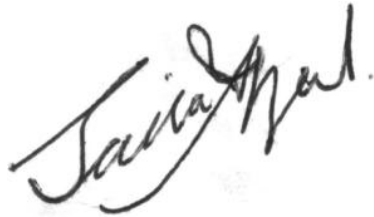

Dr. Saira Afzal Editor Annals of KEMU 